\title{
1-S06-3 Symposium6
}

\section{EAAC1 gene deletion reduces adult hippocampal neurogenesis after transient cerebral ischemia}

\author{
Bo Young Choi ${ }^{1}$, Min Sohn ${ }^{2}$, Sang Won Suh ${ }^{1}$
}

${ }^{I}$ Department of Physiology, Hallym University, College of Medicine, ${ }^{2}$ Department of Nursing, Inha University

Several studies have demonstrated that excitatory amino acid carrier-1 (EAAC1) gene deletion exacerbates hippocampal and cortical neuronal death after ischemia. However, presently there are no studies investigating the role of EAAC1 in hippocampal neurogenesis. In this study, we tested the hypothesis that reduced cysteine transport into neurons by EAAC1 knockout negatively affects adult hippocampal neurogenesis under physiological or pathological states. This study used young mice (aged 3-5 months) and aged mice (aged 11-15 months) of either the wild-type (WT) or EAAC1-/- genotype. Ischemia was induced through the occlusion of bilateral common carotid arteries for 30 minutes. Histological analysis was performed at 7 or 30 days after ischemia. We found that both young and aged mice with loss of the EAAC1 displayed unaltered cell proliferation and neuronal differentiation, as compared to agematched WT mice under ischemia-free conditions. However, neurons generated from EAAC1-/- mice showed poor survival outcomes in both young and aged mice. In addition, deletion of EAAC1 reduced the overall level of neurogenesis, including cell proliferation, differentiation, and survival after ischemia. The present study demonstrates that EAAC1 is important for the survival of newly generated neurons in the adult brain under physiological and pathological conditions. Therefore, this study suggests that EAAC1 plays an essential role in modulating hippocampal neurogenesis. 